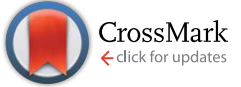

Cite this: RSC Adv., 2017, 7, 13584

Received 24th December 2016 Accepted 24th February 2017

DOI: 10.1039/c6ra28644e

rsc.li/rsc-advances

\title{
Enhanced hole injection in organic light-emitting diodes utilizing a copper iodide-doped hole injection layer
}

\begin{abstract}
Meiling Shan, Haipeng Jiang, Yu Guan, Dongsu Sun, Yu Wang, Jie Hua and Jin Wang*
We have demonstrated organic light-emitting diodes (OLEDs) by incorporating copper iodide (Cul) in $4,4^{\prime}, 4^{\prime \prime}$-tris $(N-3$-methylphenyl- $N$-phenyl-amino)triphenylamine ( $m$-MTDATA) as a hole injection layer (HIL) based on the emitting system of $\mathrm{C} 545 \mathrm{~T}-\mathrm{Alq}_{3}$. The device with Cul-doped $m$-MTDATA HIL shows a very low operating voltage of about 4.26 and $5.70 \mathrm{~V}$ for 1000 and $10000 \mathrm{~cd} \mathrm{~m}^{-2}$, respectively, which reveals a great improvement over the undoped device $m$-MTDATA HIL $\left(4.85 \mathrm{~V}\right.$ for $1000 \mathrm{~cd} \mathrm{~m}^{-2}$ and $7.72 \mathrm{~V}$ for $10000 \mathrm{~cd} \mathrm{~m}^{-2}$ ). Furthermore, the Cul-doped device exhibits a maximum power efficiency of $5.88 \mathrm{~lm} \mathrm{~W}^{-1}$, about $58 \%$ higher than that of the corresponding undoped device $\left(3.71 \mathrm{~lm} \mathrm{~W}^{-1}\right)$. The improved performance of the Cul-doped device could be attributed to the enhanced hole injection and transport due to the generation of free charge carriers in the Cul-doped layer by charge transfer from $m$-MTDATA to Cul molecules, leading to an increase in electrical conductivity and formation of ohmic contact at the ITO/HIL interface.
\end{abstract}

\section{Introduction}

Organic light-emitting diodes (OLEDs) are potential devices for application in eco-friendly next-generation displays and energysaving solid-state lighting sources due to their intrinsic properties, such as high efficiency, environmental friendliness, flexibility, and easy fabrication..$^{1-5}$ Although the performance of OLEDs has had significant advances over the past two decades, their power consumption is one of the most crucial issues for commercial applications up to date. ${ }^{6-12}$ In general, a plurality of energy barriers for charge injection is present at the electrode/ organic and organic/organic interface in OLEDs. The barrier commonly would result in high operating voltage, low efficiency, and fast degradation in OLEDs because observed currents are controlled by both charge injection and transport. $^{\mathbf{1 3 , 1 4}}$ Therefore, a low barrier at the interface is desired for efficient charge injection and is generally a prerequisite to reduce power consumption.

In the case of the anode side, the quality of the hole injection layer (HIL) in OLEDs is a crucial factor for the operating voltage, power efficiency, and stability of the device. Recently, various kinds of organic or inorganic semiconductor materials as dopants were introduced for HIL in OLEDs to reduce the hole injection barrier (HIB) at the anode/organic interface and increase hole transporting ability at the bulk. ${ }^{15}$ Specially, transition metal oxides (TMOs), including molybdenum, tungsten,

Key Laboratory of Functional Materials Physics and Chemistry of the Ministry of Education, College of Information Technology, Jilin Normal University, Siping 136000, China.E-mail: jwang@jlnu.edu.cn; Tel: +864343290834 vanadium, and rhenium oxides $\left(\mathrm{MoO}_{3}, \mathrm{WO}_{3}, \mathrm{~V}_{2} \mathrm{O}_{5}\right.$, and $\left.\mathrm{ReO}_{3}\right){ }^{16-19}$ have been doped into HIL as n-type dopants to enhance device performance. Such transition metal oxides have deep-lying energy band structures and natural oxygen vacancies. Furthermore, their conduction band edges are located very close to the Fermi level of the anode, which facilitate hole injection and transport due to the interfacial charge generation at TMO/organic interface. In addition, metal halide materials such as copper iodide (CuI), ${ }^{20}$ antimony pentachloride $\left(\mathrm{SbCl}_{5}\right),{ }^{21}$ ferric chloride $\left(\mathrm{FeCl}_{3}\right){ }^{22}$ and sodium chloride $(\mathrm{NaCl}),{ }^{23}$ have been successfully utilized as a p-type semiconductor material for improving hole injection in OLEDs. Among these materials, CuI shows high optical transparency in the visible spectral range, high work function, good conductivity, and low thermal evaporation temperature, which make this material particularly suitable for HIL. ${ }^{\mathbf{2 0 2 4}} \mathrm{Kim}$ et al. demonstrated top-emitting OLEDs using CuI doped in NPB as the HIL to enhance the hole injection from $\mathrm{Ag}$ metal anode. ${ }^{20}$ In this paper, we introduce $\mathrm{CuI}$ as the electron-acceptor dopant in $4,4^{\prime}, 4^{\prime \prime}$-tris $(\mathrm{N}-3$ methylphenyl- $N$-phenyl-amino)triphenylamine ( $m$-MTDATA) to form a p-doping HIL in OLEDs for improving the hole injection. Hole injection from the ITO anode to $m$-MTDATA is dramatically enhanced by the formation of charge transfer complex between $m$-MTDATA and CuI. The device exhibits extremely low operating voltage of about 4.26 and $5.70 \mathrm{~V}$ for 1000 and $10000 \mathrm{~cd} \mathrm{~m}^{-2}$, respectively, which are among the best reported for the device based on 2,3,6,7-tetrahydro-1,1,7,7,-tetramethyl-1H,5H,11 $H$-10-(2-benzothiazolyl)quinolizino $\quad[9,9 a, 1 g h]$ coumarin (C545T)-Alq ${ }_{3}$ system. Moreover, the CuI-doped device 
exhibits a maximum power efficiency of $5.88 \mathrm{~lm} \mathrm{~W}^{-1}$, about $58 \%$ higher than that of the corresponding undoped device.

\section{Experimental details}

The OLEDs were fabricated on glass substrates commercially precoated with a layer of indium tin oxide (ITO, a sheet resistance of $40 \Omega \mathrm{sq}^{-1}$ ) as anode. The substrates were thoroughly cleaned sequentially with acetone, alcohol and deionized water by scrubbing and sonication, then dried in an oven, and treated in an ultraviolet-ozone chamber prior to insertion into a high vacuum deposition chamber with a base pressure less than 5.0 $\times 10^{-4} \mathrm{~Pa}$. All materials used in this work were purchased from Luminescence Technology Corporation except for CuI, which was purchased from Sigma-Aldrich, and used directly without further purification. The molecular structures of the organic materials can be found elsewhere. Organic materials were deposited by thermal evaporation at the rates of $\sim 0.1 \mathrm{~nm} \mathrm{~s}^{-1}$ on an ITO patterned glass substrate. After the deposition of organic semiconducting materials, the device was completed by depositing an Al cathode on the sample at a rate of $>0.1 \mathrm{~nm} \mathrm{~s}^{-1}$. All doping concentrations reported herein are given in percentage by weight. All the thicknesses and deposition rates were monitored in situ using quartz crystal monitors during deposition. Hole-only devices (HODs) were prepared by the same procedure. The overlap between ITO anode and Al cathode was $4 \mathrm{~mm}^{2}$ as the active area size of devices. The current density-voltage $(J-V)$, luminance-voltage $(L-V)$ characteristics, and radiance of all devices were measured by an automatic system containing a constant current source (Keithley 2400 Source meter) and a calibrated spectrometer (Photo Research SpectraScan PR-655). The absorbance spectra of films were measured by means of UV-Vis spectrophotometer (UV 1700, Shimadzu). All devices were measured without encapsulation at room temperature under ambient conditions.

\section{Results and discussion}

In general, p-type dopants forms charge-transfer (CT) complex with hole transporting organic materials by charges transfer from the highest occupied molecular orbital (HOMO) of host materials to the lowest occupied molecular orbital (LUMO) of dopant molecules. ${ }^{25}$ If we consider that $m$-MTDATA possesses HOMO level of $5.1 \mathrm{eV}$ and CuI has a high work function of about $5.5 \mathrm{eV},{ }^{26} \mathrm{CT}$ complex can easily form as detected by the presence of additional absorption peaks in absorbance spectrum. ${ }^{27}$ Fig. 1 shows the UV-Vis absorbance spectra of the glass substrate/pure $m$-MTDATA film $(40 \mathrm{~nm})$ and glass substrate/CuI: $m$-MTDATA films $(5 \%, 10 \%, 20 \%$, and $33 \%)(40 \mathrm{~nm})$. The inset shows the absorbance spectrum of the $40 \mathrm{~nm}$ CuI film on glass substrate. The strong absorption peaks of both the pure $m$-MTDATA and $\mathrm{CuI}$ film are located at the wavelength less than $410 \mathrm{~nm}$, while the CuI-doped films show an additional absorption band in the range from about 410 to $490 \mathrm{~nm}$, which is attributed to the formation of CT complex owing to $\mathrm{CuI}$ accepting electrons from $m$-MTDATA. ${ }^{15,21}$ Moreover, as the CuI doping concentration increases, the additional absorption peak increases,

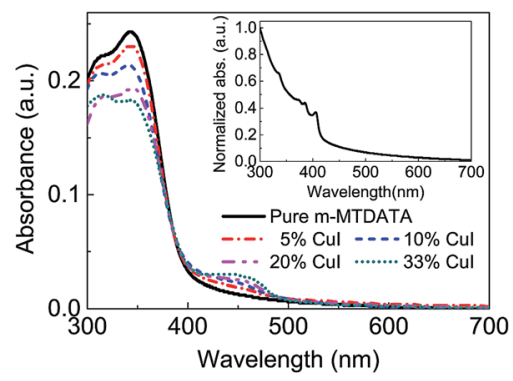

Fig. 1 Absorbance spectra of the pure m-MTDATA and Cul-doped $m$-MTDATA films at various Cul concentration. Inset: absorbance spectrum of the pure Cul film.

companying with a decrease in the original absorption peak of pure $m$-MTDATA at shorter wavelengths. This result demonstrates that the CuI molecules contribute to the formation of the CT complex until a doping concentration of $33 \%$ is reached. Similar results are also observed in CuI-doped 2T-NATA film. ${ }^{28}$ Fig. 2 and the inset show the measured current density-voltageluminance $(J-V-L)$ and current efficiency versus current density characteristics of the $\mathrm{Alq}_{3}$-based devices with the various thickness of CuI-doped $m$-MTDATA HIL, which consists of ITO/ CuI: $m$-MTDATA $(10,20$, and $30 \mathrm{~nm}) / m$-MTDATA $(30,20$, and $10 \mathrm{~nm}) / N, N^{\prime}$-bis(naphthalen-1-yl)- $N, N^{\prime}$-bis(phenyl)-benzidine (NPB) $(10 \mathrm{~nm}) /$ tris(8-hydroxy-quinolinato)aluminium $\left(\mathrm{Alq}_{3}\right)$ $(60 \mathrm{~nm}) / \mathrm{LiF}(0.8 \mathrm{~nm}) / \mathrm{Al}(120 \mathrm{~nm})$, where the CuI doping concentration was fixed at $10 \%$. For comparison, a reference device of undoped $m$-MTDATA with ITO $/ m$-MTDATA $(40 \mathrm{~nm}) /$ $\mathrm{NPB}(10 \mathrm{~nm}) / \mathrm{Alq}_{3}(60 \mathrm{~nm}) / \mathrm{LiF}(0.8 \mathrm{~nm}) / \mathrm{Al}(120 \mathrm{~nm})$ was fabricated simultaneously. Compared with the undoped device, the devices with CuI-doped $m$-MTDATA layer are able to generate a substantially larger current density at the same operating voltage. These results indicate that the CuI-doped layer would be effective for voltage reduction. For instance, the operating voltage significantly reduces from 6.37 to $5.45 \mathrm{~V}$ at $50 \mathrm{~mA} \mathrm{~cm}{ }^{-2}$ when the thickness of the doped layer increases from 0 to $20 \mathrm{~nm}$, while the operating voltage slightly reduces to $5.34 \mathrm{~V}$ as the thickness further increases to $30 \mathrm{~nm}$. It is clearly seen that hole injection and transport have been enhanced in the devices with the CuI-doped $m$-MTDATA layer. Besides, the doped devices show higher brightness than the undoped device, and

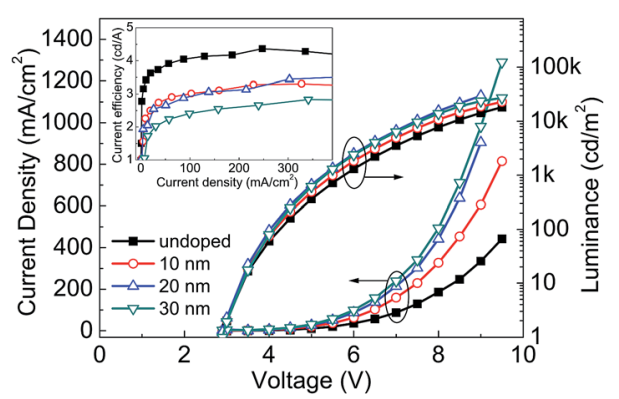

Fig. 2 The $J-V-L$ characteristics of the devices with various thickness of 10\% Cul-doped m-MTDATA HIL. Inset: current efficiency vs. current density characteristics. 
the one with CuI-doped layer thickness of $20 \mathrm{~nm}$ exhibits better luminance performance. However, it is worth noting that doping $\mathrm{CuI}$ in the $m$-MTDATA layer results in a slight decrease in the current efficiency, which mainly results from charge imbalance in the emissive layer of the OLEDs due to the enhanced hole injection and transport. ${ }^{29}$

To obtain the detailed information on the enhancement of hole injection and transport caused by doping of $\mathrm{CuI}$ in $m$-MTDATA layer, a series of HODs with ITO/HIL/NPB/Al structure were investigated. In this device geometry, the current flows proceed predominantly by the transport of hole due to the large electron injection barrier from the $\mathrm{Al}$ cathode to the NPB and the small electron mobility of the NPB. Fig. 3 shows the $J-V$ characteristics of the HODs consisting of ITO/ 10\% CuI: $m$-MTDATA $(0,10,20$, and $30 \mathrm{~nm}) / m$-MTDATA $(40,30$, 20 , and $10 \mathrm{~nm}) / \mathrm{NPB}(60 \mathrm{~nm}) / \mathrm{Al}$. Obviously, the current density of the CuI-doped HODs increases significantly at the same forward operating voltage relative to the undoped HOD. Furthermore, with the increase of the thickness of the doped layer, the operating voltage decreases until the optimum thickness is $20 \mathrm{~nm}$. However, as the doped layer further increases over $20 \mathrm{~nm}$, the device exhibits a relatively slow decrease in the operating voltage. The change in operating voltage is in agreement with that of the CuI-doped $\mathrm{Alq}_{3}$-based devices (as shown in Fig. 2). Therefore, this result reveals that the CuI-doped $m$-MTDATA indeed leads to lower operating voltage due to the enhanced hole injection and transport.

In addition, the reduction in operating voltage would result from both the interface and bulk effects of the doped layer. ${ }^{30,31}$ To further study hole injection capability of CuI-doped $m$-MTDATA layer, we fabricated a series of HODs with various CuI doping concentration, which configuration is ITO/CuI:mMTDATA $(x \%, 40 \mathrm{~nm}) / \mathrm{NPB}(60 \mathrm{~nm}) / \mathrm{Al}$, where $x$ is $0,5,10,20$, and 33, respectively. As shown in Fig. 4, the CuI-doped HODs show larger hole current than the undoped HODs, suggesting that CuI-doped $m$-MTDATA should be the better hole injection interfacial layer onto ITO anode in OLEDs. Furthermore, as the CuI doping concentration increasing, the $J-V$ characteristics of the devices gradually shifts to the lower operating voltage, indicating that the hole injection efficiency increased steadily with the CuI doping concentration increases. The observed reduction of the operating voltage can be explained based on the conductivity of the doped films. The device with structure of ITO/CuI:m-MTDATA (0\%, 5\%, 10\%, 20\%, and 33\%) (60 nm)/Al

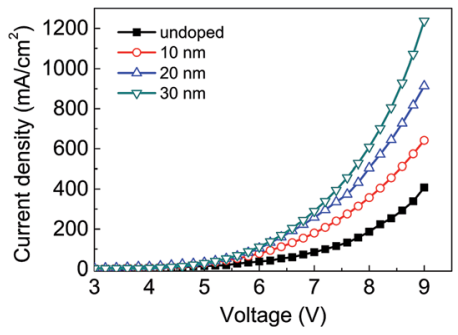

Fig. 3 The $J-V$ characteristics of the HODs with various thickness of 10\% Cul-doped $m$-MTDATA.

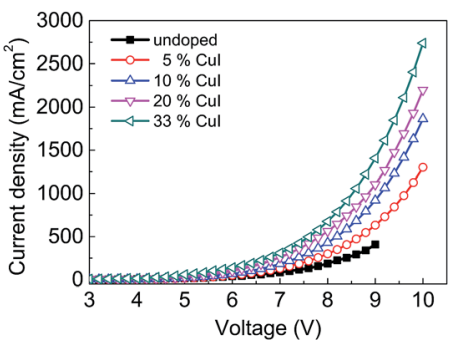

Fig. 4 The $J-V$ characteristics of the HODs with various Cul concentration.

was used to investigate the change of electrical conductivity for the different CuI concentrations in $m$-MTDATA and the $J-V$ characteristics of the devices, as shown in Fig. 5. The $J-V$ characteristics of the device with undoped $m$-MTDATA layer shows good coincidence with trapped charge limited current model (below $\sim 1.4 \mathrm{~V}$ ) and space charge limited current model (above $\sim 1.4 \mathrm{~V}$ ). In contrast, it is clearly seen that the doped

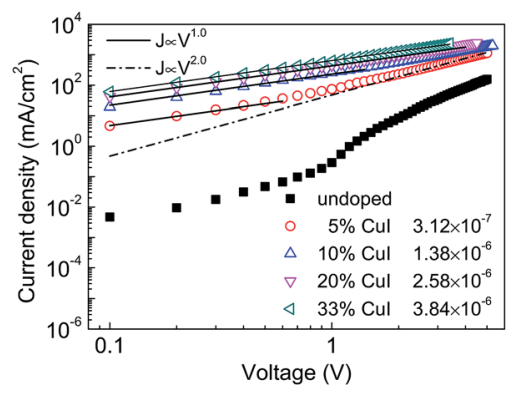

Fig. $5 \mathrm{~J}-V$ characteristics of ITO/Cul:m-MTDATA (0\%, 5\%, 10\%, 20\%, and $33 \%)(60 \mathrm{~nm}) / \mathrm{Al}$.
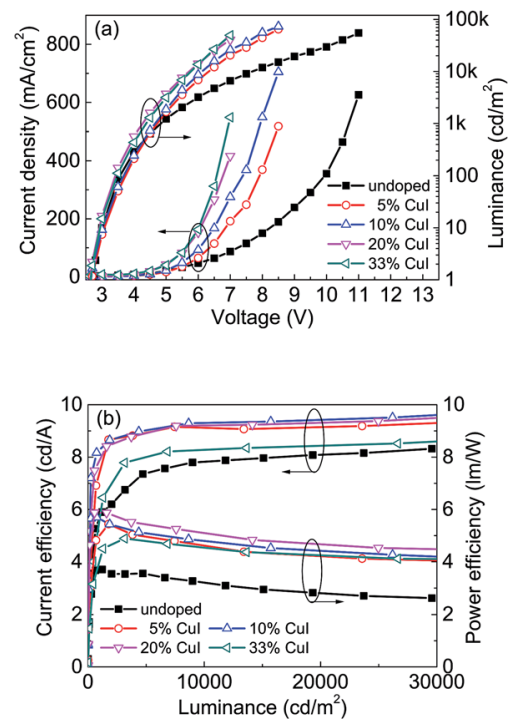

Fig. 6 (a) $J-V-L$ and (b) current efficiency-luminance-power efficiency characteristics of devices: ITO/Cul:m-MTDATA $(x \%, 20 \mathrm{~nm}) / \mathrm{m}$ MTDATA $(20 \mathrm{~nm}) / \mathrm{NPB}(10 \mathrm{~nm}) / \mathrm{C} 545 \mathrm{~T}: \mathrm{Alq}_{3}:(1 \%, 35 \mathrm{~nm}) / \mathrm{Alq}_{3}(35 \mathrm{~nm}) /$ LiF $(0.8 \mathrm{~nm}) / \mathrm{Al}(120 \mathrm{~nm})(x=0,5,10,20$, and 33). 
Table 1 Electroluminescence performance of devices with undoped and Cul-doped $m$-MTDATA HIL

\begin{tabular}{lllll}
\hline $\begin{array}{l}\text { Doping rate } \\
(\%)\end{array}$ & $\begin{array}{l}\text { Operating voltage } \\
(\mathrm{V})\end{array}$ & $\begin{array}{l}\text { Current efficiencies }^{b} \\
\left(\mathrm{~cd} \mathrm{~A}^{-1}\right)\end{array}$ & $\begin{array}{l}\text { Power efficiencies }^{b} \\
\left(\mathrm{~lm} \mathrm{~W} \mathrm{~W}^{-1}\right)\end{array}$ & $\begin{array}{l}\text { Maximum luminance } \\
\left(\mathrm{cd} \mathrm{m} \mathrm{m}^{-2}\right)\end{array}$ \\
\hline Undoped & $4.85,6.60,7.72$ & $8.64,5.63,7.36$ & $3.71,3.68,3.53$ & 54860 at 11.0 \\
5 & $4.72,5.75,6.33$ & $9.78,7.41,8.93$ & $5.44,5.00,4.96$ & 796 at 8.5 \\
10 & $4.66,5.60,6.11$ & $10.40,8.26,9.02$ & $5.70,5.63,5.08$ & 73260 at 8.5 \\
20 & $4.26,5.20,5.70$ & $9.75,7.89,8.93$ & $5.88,5.87,5.43$ & 40630 at 7.0 \\
33 & $4.38,5.29,5.77$ & $9.04,5.63,7.98$ & $4.89,4.05,4.79$ & 49550 at 7.0
\end{tabular}

${ }^{a}$ Order of measured operating voltage at 1000,5000 and $10000 \mathrm{~cd} \mathrm{~m}^{-2} \cdot{ }^{b}$ Order of measured value: maximum, then values at 1000 and $5000 \mathrm{~cd} \mathrm{~m}{ }^{-2}$.

devices exhibit ohmic-like $J-V$ characteristics $(J \propto V)$ at low voltage. The electrical conductivity calculated from quasi-ohmic region of the doped CuI devices are shown in inset of Fig. 5, which indicates the conductivity enhances with the increase of the CuI concentration in $m$-MTDATA, and its many orders of magnitude higher than that of undoped $m$-MTDATA $\left(\sim 10^{-10} \mathrm{~S}\right.$ $\mathrm{cm}^{-1}$ ). This behavior demonstrates that doping $\mathrm{CuI}$ in $\mathrm{m}^{-}$ MTDATA layer enhances the charge injection and transport due to the increase in free holes concentration in the doped layer, which is caused by the electron charge transfer from $\mathrm{m}^{-}$ MTDATA to CuI molecules. Yi et al. also recently reported that $\mathrm{CuI}$ interlayer dramatically reduced the HIB height from ITO to organic layer owing to the electron-withdrawing property of $\mathrm{CuI}$ and relatively small interface dipole, ${ }^{26}$ which may be responsible for the enhancement of the hole injection simultaneously in our work.

Since it was proved that holes could be more easily injected by CuI doped in $m$-MTDATA layer, we investigated the effect of CuI doping concentration on the device performance. The detailed device configuration was as follows: ITO/CuI:mMTDATA $(x \%, 20 \mathrm{~nm}) / m$-MTDATA $(20 \mathrm{~nm}) / \mathrm{NPB}(10 \mathrm{~nm}) /$ C545T:Alq $3(1 \%, 35 \mathrm{~nm}) / \mathrm{Alq}_{3}(35 \mathrm{~nm}) / \mathrm{LiF}(0.8 \mathrm{~nm}) / \mathrm{Al}(120 \mathrm{~nm})$, where C545T acted as dopant for green emission. In the CuIdoped layer, the doping concentration in percentages by weight is $0 \%, 5 \%, 10 \%, 20 \%$, and $33 \%$, respectively. Fig. $6(\mathrm{a})$ illustrates $J-V-L$ characteristics of the devices with various CuI doping concentration. Detailed performance parameters of devices are listed in Table 1. In good accordance with the results of HODs experiments above, the devices with CuIdoped $m$-MTDATA show lower operating voltage than the undoped ones. The undoped device exhibits operating voltage of $6.09 \mathrm{~V}$ at $50 \mathrm{~mA} \mathrm{~cm}{ }^{-2}$. In contrast, the CuI-doped devices show an obviously reduction of operating voltage. For instance, the devices with $5 \%, 10 \%, 20 \%$, and $33 \%$ CuI-doped $m$-MTDATA layer show the operating voltages of $5.74,5.50$, 5.08 , and $5.11 \mathrm{~V}$ at $50 \mathrm{~mA} \mathrm{~cm}{ }^{-2}$, respectively. In addition, at a luminance of $1000 \mathrm{~cd} \mathrm{~m}^{-2}$, the operating voltage for doped devices is in the range from 4.72 to $4.26 \mathrm{~V}$, while that for undoped device is $4.85 \mathrm{~V}$. The present device based on the emitting system of C545T-Alq ${ }_{3}$ exhibits the lowest operating voltage of $4.26 \mathrm{~V}\left(1000 \mathrm{~cd} \mathrm{~m}^{-2}\right)$ among the best ever reported in the literatures. ${ }^{32-34}$ Furthermore, from the summarized data in Table 1, it can be seen that the maximum luminance of 73260 $\mathrm{cd} \mathrm{\textrm {m } ^ { - 2 }}$ and maximum current efficiency of $10.40 \mathrm{~cd} \mathrm{~A}^{-1}$ are achieved in the $10 \%$ CuI-doped device. The current and power efficiency characteristics of the same devices are shown in Fig. 6(b). The current efficiency increases from 7.36 to $8.93 \mathrm{~cd}$ $\mathrm{A}^{-1}$ at $5000 \mathrm{~cd} \mathrm{~m}^{-2}$ with increasing of CuI doping concentration from $0 \%$ to $5 \%$. Moreover, the devices demonstrate similar current efficiency of around $9.0 \mathrm{~cd} \mathrm{~A}^{-1}$ at $5000 \mathrm{~cd} \mathrm{~m}^{-2}$ when the doping concentration varies from $5 \%$ to $20 \%$. As the concentration further increases to $33 \%$, the current efficiency decreases to $7.98 \mathrm{~cd} \mathrm{~A}^{-1}$ at $5000 \mathrm{~cd} \mathrm{~m}^{-2}$. It should be noted that the better performance in current efficiency of the doped devices originates from the improvement of hole injection and transport as well as good charge balance in the emissive layer. It is obvious that the doped devices show substantially enhanced power efficiency in comparison with the undoped device, and optimum performance is obtained in the device with doping concentration of $20 \%$. The optimized device exhibits a maximum power efficiency of $5.88 \mathrm{~lm} \mathrm{~W}^{-1}$ at about $570 \mathrm{~cd} \mathrm{~m}^{-2}$, which increases about $58 \%$ as compared to that of the undoped device owing to reduction in operating voltage by CuI doped in $m$-MTDATA layer. Remarkably, the power efficiency of optimized device slightly decreases from the maximum value of 5.88 to $5.43 \mathrm{~lm} \mathrm{~W}^{-1}\left(5000 \mathrm{~cd} \mathrm{~m}^{-2}\right.$ at $\left.5.20 \mathrm{~V}\right)$, and even at extremely high luminance of $10000 \mathrm{~cd} \mathrm{~m}^{-2}(5.70$ $\mathrm{v})$, the power efficiency can still reach $5.10 \mathrm{~lm} \mathrm{~W}^{-1}$.

\section{Conclusions}

In summary, we demonstrated efficient OLEDs with CuI-doped $m$-MTDATA HIL based on the emitting system of C545T-Alq . Compared to the undoped device, the devices with doping CuI in $m$-MTDATA layer exhibited a lower operating voltage and an enhanced power efficiency. When the doping concentration is $20 \%$, the CuI-doped device exhibited very low operating voltage of about $4.26 \mathrm{~V}$ for $1000 \mathrm{~cd} \mathrm{~m}^{-2}$ and $5.70 \mathrm{~V}$ for $10000 \mathrm{~cd} \mathrm{~m}^{-2}$. A maximum power efficiency of $5.88 \mathrm{~lm} \mathrm{~W}^{-1}$ was realized, which increased about $58 \%$ comparing with the undoped device. The improvement of device performance was ascribed to the enhanced hole injection and transport owing to the p-type doping effects in the organic films and hole concentration increases, resulting in an increase in electrical conductivity and formation of ohmic contact at the ITO/HIL interface. This result suggests that the combination of optimized thickness and concentration of CuI doping in HIL is an excellent strategy to enhance the hole injection and transport in OLEDs. 


\section{Acknowledgements}

This work was supported by the National Natural Science Foundation of China (No. 11274304) and the Natural Science Foundation of Jilin Province of China (No. 20101512, 20121521).

\section{References}

1 S. Reineke, F. Lindner, G. Schwartz, N. Seidler, K. Walzer, B. Lussem and K. Leo, White organic light-emitting diodes with fluorescent tube efficiency, Nature, 2009, 459, 234-238. 2 Y. S. Tyan, Organic light-emitting-diode lighting overview, $J$. Photonics Energy, 2011, 1, 011009-011015.

3 T. H. Han, Y. Lee, M. R. Choi, S. H. Woo, S. H. Bae, B. H. Hong, J. H. Ahn and T. W. Lee, Extremely efficient flexible organic light-emitting diodes with modified graphene anode, Nat. Photonics, 2012, 6, 105-110.

4 Q. S. Zhang, B. Li, S. P. Huang, H. Nomura, H. Tanaka and C. Adachi, Efficient blue organic light-emitting diodes employing thermally activated delayed fluorescence, Nat. Photonics, 2014, 8, 326-332.

5 Y. H. Duan, Y. Duan, X. Wang, D. Yang, Y. Q. Yang, P. Chen, F. B. Sun, K. W. Xue and Y. Zhao, Highly flexible peeled-off silver nanowire transparent anode using in organic lightemitting devices, Appl. Surf. Sci., 2015, 351, 445-450.

6 X. Zhou, J. Blochwitz, M. Pfeiffer, A. Nollau, T. Fritz and K. Leo, Enhanced hole injection into amorphous holetransport layers of organic light-emitting diodes using controlled p-type doping, Adv. Funct. Mater., 2001, 11, 310314.

7 S. A. Choulis, V. E. Choong, A. Patwardhan, M. K. Mathai and F. So, Interface modification to improve hole-injection properties in organic electronic devices, Adv. Funct. Mater., 2006, 16, 1075-1080.

8 J. F. Li, S. F. Chen, S. H. Su, K. S. Hwang and M. Yokoyama, Effects of hole injection layer thickness on the luminescent properties of white organic light-emitting diodes, Appl. Surf. Sci., 2006, 253, 2522-2524.

9 C. H. Gao, S. D. Cai, W. Gu, D. Y. Zhou, Z. K. Wang and L. S. Liao, Enhanced Hole Injection in Phosphorescent Organic Light-Emitting Diodes by Thermally Evaporating a Thin Indium Trichloride Layer, ACS Appl. Mater. Interfaces, 2012, 4, 5211-5216.

10 Z. Ma, J. Zhao, X. Wang and J. S. Yu, Effect of bulk and planar heterojunctions based charge generation layers on the performance of tandem organic light-emitting diodes, Org. Electron., 2016, 30, 136-142.

11 W. Y. Tan, D. Y. Gao, S. Zhong, J. Zhang, J. H. Zou, X. H. Zhu, W. Chen, J. B. Peng and Y. Cao, (2,2'-Binaphthyl-6,6'-diyl) bis(diphenylphosphine oxide) as a potentially simple and efficient electron-transport layer for stable organic lightemitting diodes, Org. Electron., 2016, 28, 269-274.

12 J. G. Jang, H. J. Ji, H. S. Kim and J. C. Jeong, TPBI:FIrpic organic light emitting devices with the electron transport layer of Bphen/Alq ${ }_{3}$, Curr. Appl. Phys., 2011, 11, S251-S254.
13 D. Y. Zhou, S. D. Cai, W. Gu, L. S. Liao and S. T. Lee, Efficiency dependence on alkali metal compound/Al bilayer cathode in organic light-emitting diodes, Appl. Phys. Lett., 2010, 97, 223302-223304.

14 H. Aziz, Z. D. Popovic, N. X. Hu, A. M. Hor and G. Xu, Degradation mechanism of small molecule-based organic light-emitting devices, Science, 1999, 283, 1900-1902.

15 X. Zhou, M. Pfeiffer, J. Blochwitz, A. Werner, A. Nollau, T. Fritz and K. Leo, Very-low-operating-voltage organic light-emitting diodes using a p-doped amorphous hole injection layer, Appl. Phys. Lett., 2001, 78, 410-412.

16 M. Kröger, S. Hamwi, J. Meyer, T. Riedl, W. Kowalsky and A. Kahn, Role of the deep-lying electronic states of $\mathrm{MoO}_{3}$ in the enhancement of hole-injection in organic thin films, Appl. Phys. Lett., 2009, 95, 123301-123303.

17 M. Vasilopoulou, G. Papadimitropoulos, L. C. Palilis, D. G. Georgiadou, P. Argitis, S. Kennou, I. Kostis, N. Vourdas, N. A. Stathopoulos and D. Davazoglou, High performance organic light emitting diodes using substoichiometric tungsten oxide as efficient hole injection layer, Org. Electron., 2012, 13, 796-806.

18 X. L. Zhu, J. X. Sun, H. J. Peng, Z. G. Meng, M. Wong and H. S. Kwok, Vanadium pentoxide modified polycrystalline silicon anode for active-matrix organic light-emitting diodes, Appl. Phys. Lett., 2005, 87, 153508-153510.

19 D. S. Leem, H. D. Park, J. W. Kang, J. H. Lee, J. W. Kim and J. J. Kim, Low driving voltage and high stability organic lightemitting diodes with rhenium oxide-doped hole transporting layer, Appl. Phys. Lett., 2007, 91, 011113.

20 J. H. Lee, D. S. Leem and J. J. Kim, High performance topemitting organic light-emitting diodes with copper iodidedoped hole injection layer, Org. Electron., 2008, 9, 805-808.

21 C. Ganzorig and M. Fujihira, Improved drive voltages of organic electroluminescent devices with an efficient p-type aromatic diamine hole-injection layer, Appl. Phys. Lett., 2000, 77, 4211-4213.

22 J. Endo, T. Matsumoto and J. Kido, Organic electroluminescent devices with a vacuum-deposited Lewisacid-doped hole-injecting layer, Jpn. J. Appl. Phys., 2002, 41, L358-L360.

23 J. Kim, M. Kim, J. W. Kim, Y. Yi and H. Kang, Organic light emitting diodes using NaCl: $N, N^{\prime}$-bis(naphthalene-1-yl)- $N, N^{\prime}$ bis(phenyl)benzidine composite as a hole injection buffer layer, J. Appl. Phys., 2010, 108, 103703-103707.

24 K. H. Lee, Y. S. Kwon, J. Y. Lee, S. Kang, K. S. Yook, S. O. Jeon, J. Y. Lee and S. S. Yoon, Highly Efficient Blue Organic LightEmitting Diodes Based on 2-(Diphenylamino)fluoren-7ylvinylarene Derivatives that Bear a tert-Butyl Group, Chem.-Eur. J., 2011, 17, 12994-13006.

$25 \mathrm{~W}$. Y. Gao and A. Kahn, Controlled p-doping of zinc phthalocyanine by coevaporation with tetrafluorotetracyanoquinodimethane: a direct and inverse photoemission study, Appl. Phys. Lett., 2001, 79, 4040-4042.

26 S. Park, H. Lee, J. Lee, Y. Lee and Y. Yi, Electronic structures of CuI interlayers in organic electronic devices: an interfacial studies of $N, N^{\prime}$-diphenyl- $N, N^{\prime}$-bis(1-naphthyl)-1,1'-biphenyl- 
4,4'-diamine/CuI and tris-(8-hydroxyquinolinato)aluminum/ CuI, Org. Electron., 2014, 15, 3298-3305.

27 S. Tokito, K. Noda and Y. Taga, Metal oxides as a holeinjecting layer for an organic electroluminescent device, $J$. Phys. D: Appl. Phys., 1996, 29, 2750-2753.

28 J. H. Lee, D. S. Leem, H. J. Kim and J. J. Kim, Effectiveness of p-dopants in an organic hole transporting material, Appl. Phys. Lett., 2009, 94, 123306-123308.

29 C. C. Chang, M. T. Hsieh, J. F. Chen, S. W. Hwang and C. H. Chen, Highly power efficient organic light-emitting diodes with a p-doping layer, Appl. Phys. Lett., 2006, 89, 253504-253506.

30 H. You, Y. F. Dai, Z. Q. Zhang and D. G. Ma, Improved performances of organic light-emitting diodes with metal oxide as anode buffer, J. Appl. Phys., 2007, 101, 026105026107.
31 W. J. Shin, J. Y. Lee, J. C. Kim, T. H. Yoon, T. S. Kim and O. K. Song, Bulk and interface properties of molybdenum trioxide-doped hole transporting layer in organic lightemitting diodes, Org. Electron., 2008, 9, 333-338.

32 Z. W. Liu, M. G. Helander, Z. B. Wang and Z. H. Lu, Efficient Single-Layer Organic Light-Emitting Diodes Based on C545T-Alq ${ }_{3}$ System, J. Phys. Chem. C, 2010, 114, 1193111935.

33 H. M. Zhang, Q. Fu, W. J. Zeng and D. G. Ma, High-efficiency fluorescent organic light-emitting diodes with $\mathrm{MoO}_{3}$ and PEDOT:PSS composition film as a hole injection layer, $J$. Mater. Chem. C, 2014, 2, 9620-9624.

34 W. J. Zeng, B. Ran, H. M. Zhang and W. Huang, The effect of the hole injection layer on the performance of single layer organic light-emitting diodes, J. Appl. Phys., 2014, 116, 224502-224507. 